\title{
Thermography in Neurologic Practice
}

\author{
Eduardo Borba Neves ${ }^{1, *}$, José Vilaça-Alves $^{2}$, Claudio Rosa $^{2}$ and Victor Machado Reis ${ }^{2}$ \\ ${ }^{1}$ Biomedical Engineering, Brazilian Army, Brazil \\ ${ }^{2}$ Sports Science, Trás-os-Montes e Alto Douro University, Portugal
}

\begin{abstract}
One kind of medical images that has been developed in the last decades is thermal images. These images are assessed by infrared cameras and have shown an exponential development in recent years. In this sense, the aim of this study was to describe possibilities of thermography usage in the neurologic practice. It was performed a systematic review in Web of Knowledge (Thompson Reuters), set in all databases which used two combination of keywords as "topic": "thermography" and "neurology"; and "thermography" and "neurologic". The chronological period was defined from 2000 to 2014 (the least 15 years). Among the studies included in this review, only seven were with experimental design. It is few to bring thermography as a daily tool in clinical practice. However, these studies have suggested good results. The studies of review and an analyzed patent showed that the authors consider the thermography as a diagnostic tool and they recommend its usage. It can be concluded that thermography is already used as a diagnostic and monitoring tool of patients with neuropathies, particularly in complex regional pain syndrome, and stroke. And yet, this tool has great potential for future research about its application in diagnosis of other diseases of neurological origin.
\end{abstract}

Keywords: Complex regional pain syndrome, neurologic, neurology, neuropathy, stroke, thermography.

\section{INTRODUCTION}

Medical images analysis offers a useful approach for the diagnosis and monitoring of several physical disorders. Generally, most of the tissue damage is related to changes in density, composition [1], volume and temperature of affected site [2]. The field of neurology has benefitted from several diagnostic imaging tools such as: computer tomography, Xray, nuclear medicine, ultrasound test, and nuclear magnetic resonance spectroscopy [3, 4].

One kind of medical images that has been developed in the last decades is the thermal images [5]. These images are assessed by infrared cameras and have shown an exponential development in recent years. Thermal images can show little changes in skin temperature. These changes can be related with local physiological process or systemic responses [6, 7]. It can be found studies on thermography applied to: cancer diagnosis [8], muscle damage diagnosis [6,7], diagnosis of ischemia in diabetic patients [9] and evaluation of muscle recovery [10].

Although recurrently using this tool in some areas of medicine, few studies have been found on the application of it to the neurology field. In this sense, the aim of this study was to describe possibilities of thermography usage in the neurologic practice.

*Address correspondence to this author at the Rua Dom Pedro de Menezes 25 R/C direito, Vila Real, Portugal. CEP: 5000-688; Cell: +351 910412617; Tel: +351 259350877; E-mail: borbaneves@ hotmail.com

\section{METHODS}

It was performed a systematic review in Web of Knowledge (Thompson Reuters), set in all databases which used two combination of keywords as "topic": "thermography" and "neurology"; and "thermography" and "neurologic". The chronological period was defined from 2000 to 2014 (the least 15 years). Older studies were not considered because the equipments for acquisition of thermographic images before 2000 had low sensitivity of the detectors and some technical limitations. The search was performed at November $13^{\text {th }} 2014$ and the protocol is illustrated in Fig. (1). From each study was extracted the authors/year, source of publication, study design, physical disorder studied and main findings of the study.

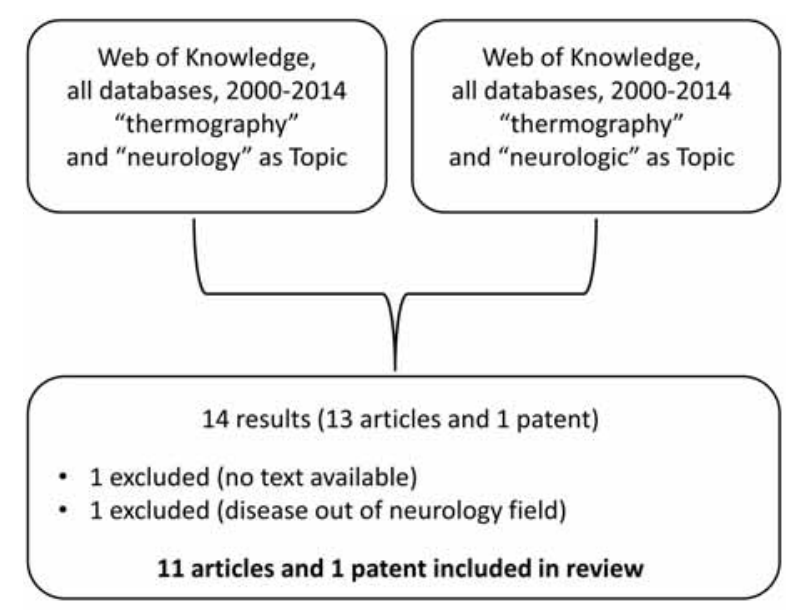

Fig. (1). Illustration of search protocol. 


\section{RESULTS}

After research, some data was extracted from articles to show the main characteristics of analyzed papers. These data are presented in Table $\mathbf{1}$.

\section{DISCUSSION}

The studies involving thermography in neurology, in this century, started in 2001 with Riedl et al. [11] that performed a research about the complex regional pain syndrome that is a special form of neuropathic pain that develops after a minor trauma in extremities. In this study participated 17 stroke patients, 21 patients in the acute and late stage of complex regional pain syndrome I (CRPS I) and 23 healthy subjects as control group. The authors performed a detailed neurological examination of the sample; after a sweating was induced peripherally and centrally by carbachol iontophoresis and quantified by evaporation hygrometry. They used the infrared thermography to assess the skin temperature. In conclusion, they suggest that many clinical symptoms in CRPS I could be explained by a central nervous system pathophysiology [11]. In this study, thermal images were used to monitoring clinical conditions of patients.

Soldo-Butkovic et al. [12], in 2002, performed the characterization of thermal patterns in neurologically normal subjects and patients with affection of peripheral neural structures of the lumbosacral region, with special reference to anterior femoral surface. The authors concluded that was an isothermal disorganization and altered the angle of inclination of isothermal lines against horizontal line and in the presence of isothermia it can be a reliable sign of

Table 1. Main characteristics of analyzed papers involving Thermography and Neurology.

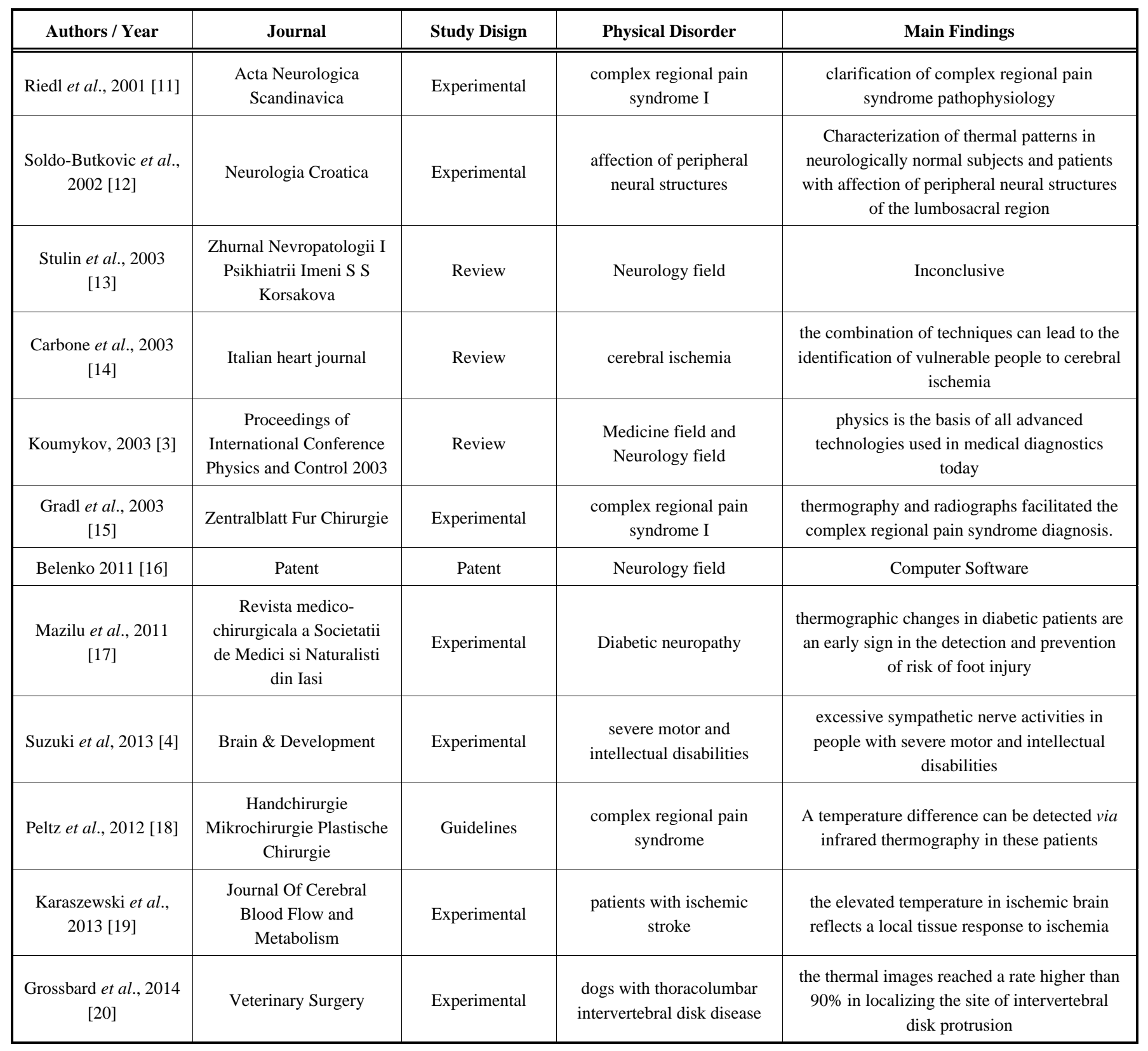


peripheral nervous system lesion at this specific localization. They proposed that this diagnostic criterion can be introduced in the clinical practice [12].

In 2003, Stulin et al. [13] published a review study which discusses the usability of thermography in neurology field, but they did not close the subject. They also reported the use of thermal images in recognizing pre-cancer and cancer conditions in mammaria, joint diseases, and diagnosis of arterial occlusion. In the same year, Carbone et al. [14] wrote another review which focused on new possibilities of investigation and detection of atherosclerotic carotid plaque and cited the intravascular thermography as one of them. This can be considered important to neurology practice because the distal embolization of atheromatous debris originated from carotid plaque disruption are the most common pathogenic mechanisms for cerebral ischemia [14]. The authors concluded that the combination of techniques can lead to the identification of vulnerable people to cerebral ischemia [14].

During the International Conference of Physics and Control in 2003, Koumykov [3] presented a paper that showed the analysis of diagnostic methods based on physical phenomena: X-ray examination, thermography, nuclear medicine, ultrasound test, endoscopy, the method of electronic paramagnetic resonance, electrocardiography, nuclear magnetic resonance spectroscopy, X-ray and positron-emission computer tomography, biomagnetism, and luminescent analysis. He justified this presentation saying that one of the actual problems of modem medicine is monitoring and control of functioning of human organs and physiological systems. Regarding the use of thermography, this explanation is in agreement with the investigations of Stulin et al. [13], Soldo-Butkovic et al. [12] and Riedl et al. [11].

The complex regional pain syndrome I represents a frequent complication following distal radial fractures [15]. Thus, Gradl et al. [15] used thermography and bilateral radiographs of both hands, apart from a detailed clinical examination during 8 and 16 weeks after trauma, to monitoring 158 patients with distal radial fractures. At the end of the observation period $11 \%$ of patients were identified as CRPS I. Thermography showed $58 \%$ of sensibility and $66 \%$ of specificity. Again, thermography was used as a complementary tool for neural disorder diagnosis.

In 2011, Belenko [16] patented his invention applied to neurology and physiology, which intended to deciphering infrared irradiation from human body surface by method of computer thermography. He used as reference a point serve infrared irradiation, with known temperature. The software can classify the regions according to temperature variations in: physiological strain, light degree decrease / enhancing of lability, medium degree decrease /enhancing of lability, or high degree decrease /enhancing of lability [16].

Diabetes mellitus is known as the most common cause of neuropathy worldwide, In this sense, Mazilu et al. [17] performed a study to demonstrate the effectiveness of the thermography method in assessing and diagnosing peripheral polyneuropathy and risk of developing ulcerative lesions in diabetic patients. This study included 90 patients of both sexes, aged from 18 to 70 years. The authors concluded that thermographic changes in patients with sensory-motor neuropathy are an early sign in the detection and prevention of risk of foot injury. So, they suggested thermography usage as a method for diagnosis and dynamic evaluation of patients with peripheral sensory-motor polyneuropathy [17].

Patients with severe motor and intellectual disabilities (SMID) frequently suffer from autonomic nervous system disorders. Therefore, early recognition of these symptoms is important to improve support for SMID. Suzuki et al. [4] that investigated the autonomic nervous function in people with SMID by skin vasomotor responses to cold stimuli. Infrared thermography was used to measure their hand skin temperature before and after cold stimuli. A distal-dorsal difference (DDD) at baseline and the recovery rate of the second fingertip and dorsum were calculated. The results suggest excessive sympathetic nerve activities in people with SMID [4].

Another document about CRPS was published by Peltz et al. [18] under the title "Diagnostic Guidelines for Complex Regional Pain Syndrome". In this document, they cited again the usage of thermography to reach a diagnostic of CRPS. He wrote that a gold standard in diagnosing CRPS has not been found, but a temperature difference that can be detected via infrared thermography and electrophysiological examinations can show a nerve lesion and support a differential diagnostic between CRPS type I and II [18].

According to Karaszewski et al. [19], pyrexia soon after stroke is associated with poor functional outcome and severe stroke. In this sense, they compared brain temperature measured twice in the first week after the stroke with tympanic temperature measurements (assessed by sensors of infrared) to determine the relationship between temperature in ischemic and contralateral normal brain with body temperature profile. Forty patients participated in this study and the authors [19] concluded that in this sample the brain temperature was higher than the body temperature; that while pyrexia reflects the systemic response to stroke, it occurs later, and is associated with adverse outcomes. The elevated temperature in ischemic brain reflects a local tissue response to ischemia.

The same authors [19] also discussed the practical applying of this knowledge writing that higher temperatures accelerate ischemic damage and opening of the blood-brain barrier. Thus, higher temperatures in contralateral brain could influence lesion progression and worsen outcome by the opening blood-brain barrier in the ischemic tissue, exacerbating tissue swelling and promoting lesion expansion. Therefore, therapeutic hypothermia might work by cooling non ischemic tissue, preventing the opening of the bloodbrain barrier, and preventing infarct growth.

A recent study [20] showed the application of thermography in the diagnosis of dogs with thoracolumbar intervertebral disk disease (TLIVDD). The sample was composed by 58 chondrodystrophic dogs with Type I TLIVDD and 14 chondrodystrophic dogs with no evidence of TLIVDD. As conclusions, the thermal images reached a rate of $90 \%$ successful differentiating among normal dogs and $97 \%$ successful in identifying the abnormal 
intervertebral disc space (localizing the site of intervertebral disk protrusion) in dogs with TLIVDD [20].

Among the studies included in this review, only seven were with experimental design. It is few to bring thermography as a daily tool in clinical practice. However, these studies $[11,15,17,18]$ have suggested good results, particularly in neuropathy as complex regional pain syndrome and diabetic neuropathy. The studies of review $[3,14,18]$ and the analyzed patent [16] showed that the authors consider the thermography as a diagnostic tool and they recommend its usage. In fact, more studies involving thermography in the field of neurology are needed.

\section{CONCLUSION}

It can be concluded that several authors recommend the use of thermography in the clinical practice of neurology, and it is already used as a diagnostic and monitoring tool of patients with neuropathies, particularly in complex regional pain syndrome, and stroke. And yet, this tool has great potential for future research about its application in diagnosis of other diseases of neurological origin. Maybe, in next years neurologists will make use of this important tool for diagnosis and monitoring clinical condition of patients.

\section{CONFLICT OF INTEREST}

The authors confirm that this article content has no conflicts of interest.

\section{ACKNOWLEDGEMENTS}

We would like to thank Brazilian Army and CNPq for important funding and financial support.

\section{REFERENCES}

[1] Neves EB, Pino AV, de Almeida RMVR, de Souza MN. Knee bioelectric impedance assessment in healthy/with osteoarthritis subjects. Physiol Measur 2010; 31: 207-19.

[2] De Meira LF, Krueger E, Neves EB, Nohama P, de Souza MA. Termografia na área biomédica. Pan Am J Med Therm 2014; 1: 3141.

[3] Koumykov VK. Physical control and monitoring in modern medicine. In: Soc ICS, Ed. Proceedings of International Conference Physics and Control. 1. St Petersburg, Russia: IEEE 2003; pp. 30812.

[4] Suzuki Y, Kobayashi M, Kuwabara K, Kawabe M, Kikuchi C, Fukuda M. Skin temperature responses to cold stress in patients with severe motor and intellectual disabilities. Brain Develop 2013; 35: 265-9.

[5] Sanches IJ, Gamba HR, de Souza MA, Neves EB, Nohama P. Fusão 3D de imagens de MRI/CT e termografia. Rev Bras Eng Bioméd 2013; 29: 298-308.

[6] Bandeira F, Neves EB, Moura MAMd, Nohama P. A termografia no apoio ao diagnóstico de lesão muscular no esporte. Rev Bras Med Esporte 2014; 20: 59-64.

[7] Bandeira F, Moura MAMd, Souza MAd, Nohama P, Neves EB. Pode a termografia auxiliar no diagnóstico de lesões musculares em atletas de futebol? Rev Bras Med Esporte 2012; 18: 246-51.

[8] Suganthi SS, Ramakrishnan S. Analysis of breast thermograms using gabor wavelet anisotropy index. J Med Sys 2014; 38:1-7.

[9] van Netten JJ, van Baal JG, Liu C, van der Heijden F, Bus SA. Infrared thermal imaging for automated detection of diabetic foot complications. J Diab Sci Technol 2013; 7: 1122-9.

[10] Neves EB, Moreira TR, Lemos RJCAd, Vilaça-Alves J, Reis VM. The Thermal Response of Biceps Brachii to Strength Training. Gazzetta Medica Italiana Archivio per le Scienze Mediche 2015; 174: (In Press).

[11] Riedl B, Beckmann T, Neundorfer B, Handwerker HO, Birklein F. Autonomic failure after stroke - is it indicative for pathophysiology of complex regional pain syndrome? Acta Neurol Scand 2001; 103: 27-34.

[12] Soldo-Butkovic S, Petravic D, Alvir D, Unusic L, Zurak N. Femoral isothermal pattern in peripheral nervous system lesions of lumbosacral localization. Neurol Croatica 2002; 51: 183-98.

[13] Stulin ID, Mnushkin AO, Musin RS, et al. Is it possible to reanimate the dying interest to the thermovision in neurology? Zhurnal Nevropatologii I Psikhiatrii Imeni S S Korsakova 2003; $8: 1-15$.

[14] Carbone GL, Mauriello A, Christiansen M, et al. Unstable carotid plaque: biochemical and cellular marker of vulnerability. Ital Heart J Suppl 2003; 4: 398-406.

[15] Gradl G, Steinborn M, Wizgall I, Mittlmeier T, Schurmann M. Acute CRPS I (Morbus sudeck) following distal radial fractures Methods for early diagnosis. Zentralblatt Fur Chirurgie 2003; 128: 1020-6.

[16] Belenko AI, inventor; Belenko AI, assignee. Method of deciphering infrared irradiation from human body surface by method of computer thermography patent RU2435522-C2; RU2009119365-A.

[17] Mazilu G, Filos C, Popescu CD. Cutaneous thermographic changes in diabetic polyneuropathy. Revista medico-chirurgicala a Societatii de Medici si Naturalisti din Iasi 2011; 115: 1007-11.

[18] Peltz E, Seifert F, Maihoefner C. Diagnostic Guidelines for Complex Regional Pain Syndrome. Handchirurgie Mikrochirurgie Plastische Chirurgie 2012; 44: 135-41.

[19] Karaszewski B, Carpenter TK, Thomas RGR, et al. Relationships between brain and body temperature, clinical and imaging outcomes after ischemic stroke. J Cerebral Blood Flow Metabol 2013; 33: 1083-9.

[20] Grossbard BP, Loughin CA, Marino DJ, et al. Medical infrared imaging (thermography) of type i thoracolumbar disk disease in chondrodystrophic dogs. Veterinary Surgery 2014; 43: 869-76. 